\title{
IL TERREMOTO DI TERAMO DEL 29 GENNAIO 1943
}

\author{
Donfenco D) Filapen
}

Volizie macrosismirle. ..- I Toral del Gran Sasoo, nella zona di ronfune tra le quallra provineie di Teramo. L'Aquila, Rieli ed Amoli P'iceno, il 29 gennato 1943 alle ore 4 a 23 minuti cil. T.M.E.C..

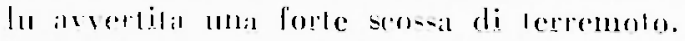

Daile nolizie macro-imiche pervenute all'Llficio Centrale di Metereologia ho potuto rilevare rhe Arpuala dal Tronto (A-roli P'ireno).

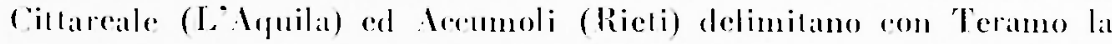

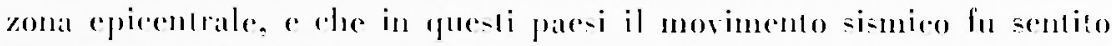
da pua-i lutli i ritadini. comprea i domienti. La popolazione delle

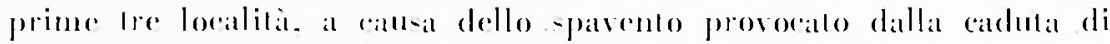
raleinared oltre ehe da qualehe leggera lesione al fabbricali. si rifugio in aperta rampagnat. Fu inothe udito. anele a Teramo, mombo con-

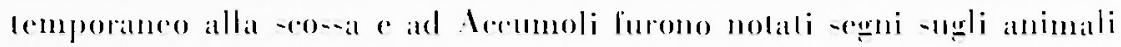
rome gualili di ranti o cauli di galli.

sureesisamente furono arrertile allere seosere allehe ene alreomfallnale da rombia. A Teramo poi fu notalo un soffio di arial caldia dellat durala di 3 mimuli rirreis. Il erremolo in que-la zona lu vallutalo di V-VT errado dellal -ailal Merralli, imeere di IV Erate alf'Armila, a Norria. Ascoli Piceno. Foligno. Pogergo Mirlelo. Notare. -ro. Cilladurale, Montallo. dove lu arrertila da mole perone de- Le con tremolio di velrale e di piecoli ogrestli. Di III grardo poi fu-entito ad Aneona. Osimo. Macorala. Cammerino. Fermo, Farla Sabina, Cilladucale e di 11 grate do at Subiaro.

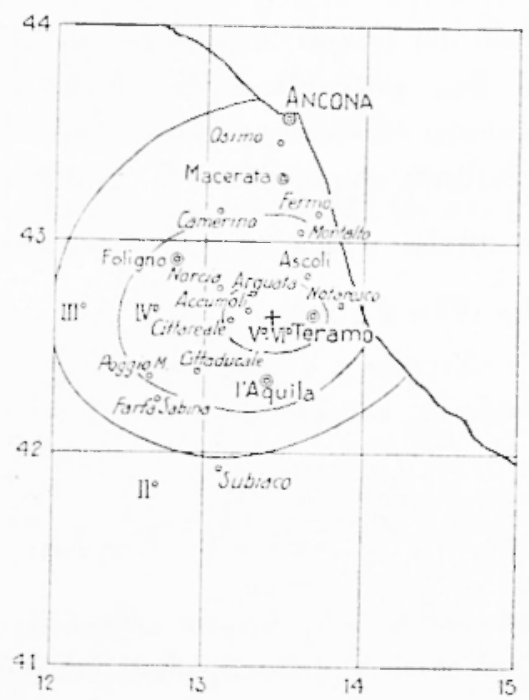

Fig. 1 
Nella cartina del luogo riprodolta nella fig. 1. sono segnale le $i=0$ -i-le, dalle cuali è possibile rilevare la regolarilí dello sposimento del movimento e la relativamente grande zona inleressala macrosismi'aimente.

Dal punto di visla sismiro la zona é molto interessante, oltre che per la presenza dei masiere monlani anche perche si sono aruli epirentri di lerremoli disastrosissimi, come quelli di Areszmo del 13 gemnaio 1915, Sinigallia, 30 oltobre 1930, Tamal dei Peligni, alle falde dellat Maiclat, 26 sellembre 1933, olte terremoli minori rome quello di Arequala del Tronto (VI) del 7 aprile 1930: e lutli hamno presenlalo una nolevole calensione macrosimical.

Determinazione dell'epientro. - Per la delerminazione dell'epicentro con metodo microsismieo ho avulo al disposizione $\mathrm{i}$ sismogrammi delle Stazioni della rele dell'Islituto Nazionale di Geofi-iea, olle a qualche allro di Oservalori nazionali e svizzeri in numero diserelo. tenuto conto delle difficolti det momento. Non ho potulo usufruire della oltinar registrazione di Camerino a rausa della mancanzal dell'intertuzione del tempo. Dato il materiale a disposizione il metodo più renveniente per la delerminazione delle coordinate epiecntrali mi è sembralo quello analitico del prof. Caloi, dhe si serve della diflerenza dei tempi di atrivo delle onde longiludinali e mascresali dirente

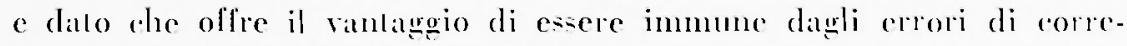
zione del tempo. Esso b basalo sulla determinazione del punto comune degli asi radieali dei cerchi di raggio $\lambda_{h}=K T_{j}$, con la mperficie

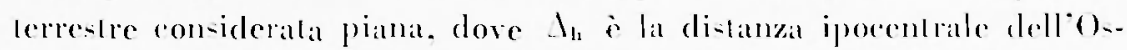
servatorio considerato. $T_{\mathrm{j}}$ la diferenza dei lempi di arrivo delle onde diretle e $K=\frac{V_{1} V_{2}}{V_{1}-V_{2}}$ essendo $V_{1}$ e $V_{2,}$, rispeltivamente, le relocili delle $P_{g}$ e Sg.

Arendo a disposizione i dali di t. o piò Stazioni, le equazioni da risolvere, considerando tutle le Stazioni allat slessa tregual cono:

$$
x_{0}+\frac{y_{\mathrm{j}}-y_{\mathrm{j}-1}}{x_{\mathrm{j}}-x_{\mathrm{j}-1}}-\frac{C_{\mathrm{i}}-C_{\mathrm{j}-1}}{x_{\mathrm{j}}-x_{\mathrm{j}-1}}=0,(i-2.3 .4 \ldots, n)
$$

dove $x_{11}, y_{11}$ e $x_{\mathrm{j}}, y_{\mathrm{j}}$ : sono ri-petlivamente le coordinate ortogonali dellepicentro e delle Stazioni considerate, e

$$
2 C_{\mathrm{j}}=x_{\mathrm{j}}^{2}+y_{\mathrm{j}}{ }^{2}-\left(K T_{\mathrm{j}}\right)^{2}(j=2,3, \ldots, n)
$$


Se si ponc

$$
u=\frac{y_{\mathrm{j}}-y_{\mathrm{j}}}{x_{\mathrm{j}}-x_{\mathrm{j}-1}}, \mathrm{i}=\frac{r_{j}-r_{j-1}}{x_{\mathrm{j}}-x_{\mathrm{j}-1}}(j=2,3, \ldots, n \mathrm{n}
$$

-i ha un -i-lema di equizioni, ehe ri-olfo col melodo dei minimi quat drati, rondure alle -enuenti equasioni normali

$$
\begin{aligned}
& |1.1| x_{0}+\left|1.01 y_{0}=\right| 1 . \rho \mid \\
& |1.11| x_{0}+\left|1.01 y_{0}=\right| 1.6|| .
\end{aligned}
$$

Po-lo come origine degli assi calrle-iani il punto di roordinate weoErafiehe, $13^{\prime \prime}>$ e 13" lis c consideralo come a-se delle y il meridiano

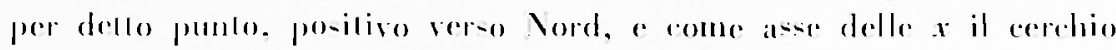

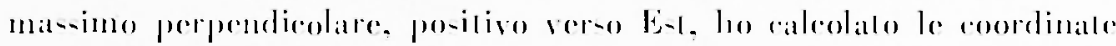

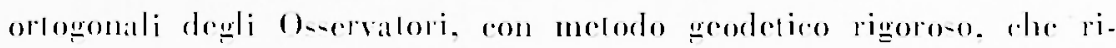
pollo

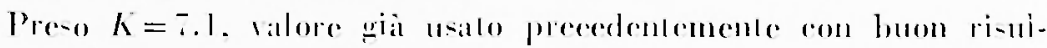
lalo per l'Tlalial Centrale. ho calcolallo i coeffirienti a e pi ollenendo

\begin{tabular}{|c|c|c|c|c|c|c|}
\hline B' & $x_{j}$ & $y_{j}$ & $T_{j}$ & $\left(\boldsymbol{K} T_{\mathrm{j}}\right)^{*}$ & "1: & li \\
\hline i iouli & Then & $.2 .208()$. & $11 . .$. & 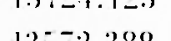 & $1400^{\circ}$ & 1 \\
\hline . & 100.0 .39 & $-3 \%, 00$ & $20-$ & - & -11.011)$. & .0 .901 \\
\hline lirenze & -110.816 & 88.5113 & 29.7 & $1+166.15=$ & -1.320 & $8 \vec{s} .132$ \\
\hline Bolognal & -132.919 & 160.575 & 36.6 & 07.527 .220 & 9.883 & -336.716 \\
\hline
\end{tabular}
per ognuma delle qualleo Stazioni considerale i valori a fianeo segnati nell al seguchle labliellat:

Sostituendo i salori di ue e nolle equazioni nommali. loo otlemulo il -i-lemil:

$$
\begin{aligned}
& 3 x_{11} \quad-7.5+1 x_{11}=-17.6 .021 \\
& 7.5 .4 x_{11}+100.077 x_{11}=-3.502 .175 .
\end{aligned}
$$

I valori delle ineognile che lo soddi-fino:

$$
x_{u}=10.311 \mathrm{~km} \quad . \quad y_{10}=-33,123 \mathrm{~km}
$$

sono le eoordinate ortogonali dellepientro. le quali. Iratormate ron melodo geodetion in roordinalle geogralfehe risultano:

$$
4 .=42 " 39^{\prime}-201^{\prime \prime} .7>K_{01}=13 " .29^{\prime} 31^{\prime \prime} .4 \mathrm{~F}
$$


Dromocrone. - Ottenule le coordinate dellepientro ho calcolato le distanze epicentrali con la nola formula di trigonometria serieal

$$
\cos \Lambda=\operatorname{sen} \varphi_{0} \operatorname{sen}\left(\varphi_{1}+\cos \varphi_{0} \cos \varphi \cos \left(\lambda_{0}-\lambda_{0}\right)\right.
$$

I ralori olfenuli per ogni osservalorio sono segnali a fimeo di ogni nominalivo nelledenco seguenle, con i lempi di arrivo dei vari impulsi riferiti al tempo medio Europa centrale e lointerpretazions delle fasi.

Considerando questi lempi ed assumendo come lempo origine provisorio $f^{\mathrm{h}} 23^{\mathrm{m}}$, ho calcolato le dromocrone delle onde tratsersali e longitudinali dietle e rifrate il cui grafieo riporto nella fiw. 2.

\begin{tabular}{|c|c|c|c|c|c|c|c|c|c|c|}
\hline$I^{\prime \prime}$ & Roma I. & G. & $1=11$ & 6,0171 & km & & $\mathrm{i}_{Z} \mathrm{P}_{\ddot{*}}$ & & & 37.9 \\
\hline & $\mathrm{i}_{Z} \mathrm{Pg}_{g}$ & $4^{h}$ & $23^{\mathrm{m}}$ & 8.9 & & & $i_{E}>n$ & & & 58.3 \\
\hline & $i_{E} S \underline{y}$ & & & 25.4 & & & $i_{N} S y$ & & 21 & 14.5 \\
\hline $2^{\prime \prime}$ & " Nappoli & & $1=21$ & 2.414 & $k m$ & $6^{\prime \prime}$ & Padoral & & $\Delta=: 3: 3$ & 1.389 \\
\hline & $\mathrm{i}_{\mathrm{N}} \mathrm{P}_{\mathrm{n}}$ & $t^{11}$ & $23^{m}$ & 23.6 & & & $\mathrm{i}_{\mathrm{N}} \mathrm{Pn}_{\mathrm{n}}$ & $4^{h}$ & $23^{111}$ & 38 \\
\hline & 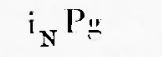 & & & 26.8 & & & $\mathrm{i}_{\mathrm{N}} P_{0}=$ & & & 19 \\
\hline & $\mathrm{i}_{\mathrm{N}}$ & & & 29 & & & & & 2.1 & 01.1 \\
\hline & $\mathrm{i}_{\mathrm{E}} S_{11}$ & & & 47 & & & $i_{x}>11$ & & & 14 \\
\hline & $i_{E} S \underline{w}$ & & & 56.2 & & $7^{0}$ & Trie-e & & $1=3$ & 1.999 \\
\hline $3^{\prime \prime}$ & ' Firenze & & $1=20$ & 0.589 & kin & & $i_{N} P_{n}$ & $f^{\prime \prime}$ & $23^{111}$ & 38.2 \\
\hline & ${ }_{\mathrm{i}} \mathrm{P}_{11}$ & $4^{\prime \prime}$ & $2 \cdot 3^{111}$ & 2.1 & & & $i_{N} P_{y}$ & & & 19,3 \\
\hline & $\mathrm{i}_{Z} P_{\underline{m}}$ & & & 28 & & & $\mathrm{i}_{\mathrm{E}} \mathrm{Sn}$ & & $24^{11}$ & 11.2 \\
\hline & $i_{N} S_{n}$ & & & 49 & & & 'EW & & & $3: 2$ \\
\hline & $i_{N} S_{\underline{r}}$ & & & 57,7 & & $8^{\circ}$ & Coira & & $1=5 t$ & $0,-7.11$ \\
\hline $4^{n}$ & "Pralo & & $1=2 ?$ & 7,333 & $k m$ & & e Pn & $4^{h}$ & 2 & 0.5 .7 \\
\hline & ${ }^{i} P_{n}$ & $f^{1}$ & $23^{11}$ & 25 & & $9^{\circ}$ & /migro & & $\lambda=6$ & $9.8 \cdots 1$ \\
\hline & $i_{N} P_{: !}$ & & & 31.3 & & & ${ }^{2} P_{n}$ & $4^{\prime \prime}$ & $2 f^{111}$ & 15.1 \\
\hline & $i_{E} S_{n}$ & & $\neg 1$ & 53,7 & & $10^{n}$ & Vinchâal & & $1=7$ & $7,+110$ \\
\hline & ${ }^{n} 2$ & & -4 & $11-1$ & & & ${ }_{N} P_{n}$ & $f^{1 / \mathrm{t}}$ & $2 \cdot 1^{111}$ & 2.2 .3 \\
\hline 511 & Bolognal & $f^{1 t}$ & $\begin{array}{l}A=2( \\
\quad ? 3^{n}\end{array}$ & $\begin{array}{c}7.811 \\
.9\end{array}$ & kIII & $11^{\circ}$ & Basilea & & $1=7$ & $3,2.22$ \\
\hline & & & & & & & c $P_{n}$ & $f^{1 /}$ & 24,11 & 24.7 \\
\hline
\end{tabular}

Onde Pg. - Parlieolarmente vistosi is slata la registrazione a Roma di questo tipo d'onda, ed anche nelle altre tazioni abbastanza 
chiare. Con i lempi rexi-trati nerli (bervalori di Romat. Napoli. Firenze, Prato, Bologrial. Padova e Triese, ho ralcolialo col metodo dei minimi quadrati lecpuazione della dromoeronta piò probabile riferilat alliperentro:

$$
t_{P_{15}}=\frac{1}{5,33}-13.01
$$

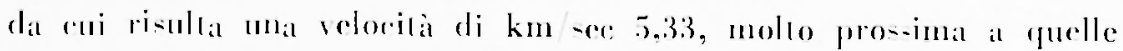
ollemule da altri riecenatori per l'I lalia Centrale e per lo - te-so lipo di onda. Le differenze la i lempi onervati e efuelli ralcolati -ono risullali:

\begin{tabular}{|c|c|c|c|}
\hline & $l_{1},-1_{1}$ & & $I_{0}-I_{\mathrm{s}}$ \\
\hline iiomil & 0,1 & Bolownal & 0.7 \\
\hline Nipoli & $-11,1$ & Pirloval & -0.2 \\
\hline Firenze & -0.4 & Tricele & 0.11 \\
\hline Pralo & -0.2 & & \\
\hline
\end{tabular}

Onde Sg - Con i lempi rilevali dai -i-mog̣rammi dei medesimi oservalori adoperali per le onde Per. erlu-o Piddoval. bo ollenulo lat

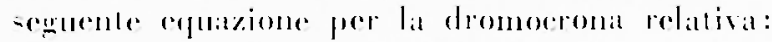

$$
t_{\mathrm{sg}_{\mathrm{g}}}=\frac{1}{3.2}-10,53
$$

\begin{tabular}{|c|c|c|c|}
\hline & $t_{0}-t_{1}$. & & $I_{0}--I_{\mathrm{r}}$ \\
\hline Romil & -0.3 & P': & 11.1 \\
\hline Nipoli & 11.3 & Bolowna & 1.2 \\
\hline pilenze & -11.7 & Triese & --1.11 \\
\hline
\end{tabular}

l.e diflerenze dei tempi sono:

Lal velocilì di que-lo li. for di ondal ir risultilia rome -i vele di 3,2 km -ree, di valore legerermente inferiore al quelli ralcolati per felaropa Centrale. mal alefuanto superiore al 3.02 kin - ore ollemulo pere Tlialia Cintrale.

Onde Pn - Lil matygion palle delle reni-lriazioni iniziano ron que-lo lipo di onclis, elar is risuliato dormegue molto

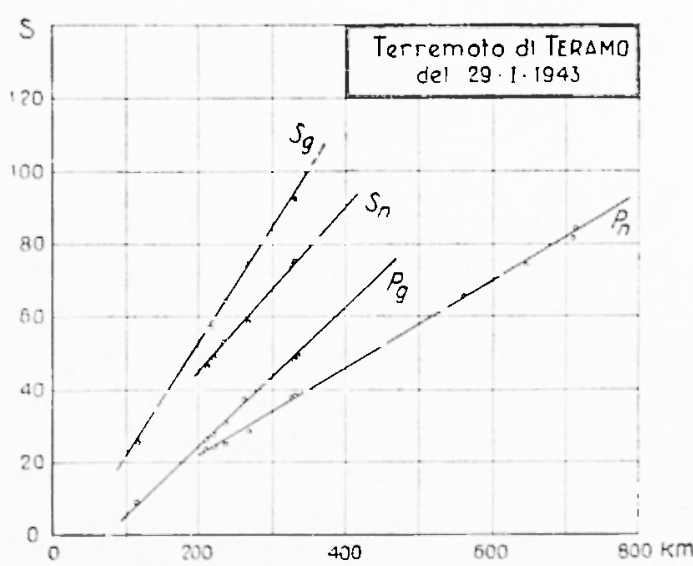

rig. 
chialro. La dromoenona piì probabile calcolala ron i tempi rilevali dai -i-mogrammi di Napoli. Firenze, Pralo, Bologna, Padora, Triese. Zu-

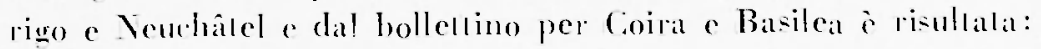

$$
t_{\mathrm{Pn}}=\frac{1}{8.25}-2,72
$$

con i seguenti seostamenti lra $t_{\text {, }}$ e $t_{\mathrm{c}}$.

\begin{tabular}{|c|c|c|c|}
\hline & $I_{n}-I_{4}$ & & $t_{0}-t_{0}$ \\
\hline Napoli & 0,2 & linesle & 0.7 \\
\hline Firenze & 0,0 & Coirar & 0,5 \\
\hline Prillo & $-1,0$ & Zurigo & -0.9 \\
\hline Bolognial & $-0,7$ & Neuchâtcl & 0,7 \\
\hline Padova & 0.6 & Basilea & 1,0 \\
\hline
\end{tabular}

Comsiderando poi le sole sazioni italiane. ho ollenuto la seguente equitzione:

$$
l_{1: 1}=\frac{1}{788}-4,22
$$

Dalle equazioni delle due dromorone sj osserva ehe eon i tempi delle slazioni ilaliane il valore ollenulo di $7,88 \mathrm{~km} /=$ per lat relociti

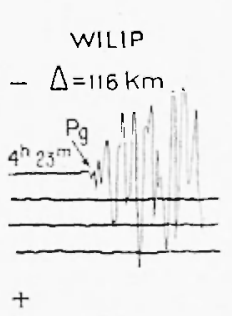

E

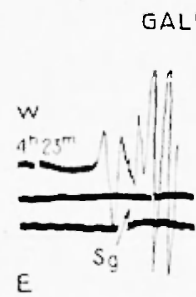

Fis. 3 di propatgakione is lewpermente inferiore a quello generalmente aceellato di $8 \mathrm{~km}$. mentre ronsiderando anche i lempi di arrivo delle slazioni. -rizzere il valore ralcolitto di $8,2.5 \mathrm{~km} / \mathrm{s}$ risulta superiore. Quteslo risultalo permelle di notale ancolal mal volta, come ebbe a rilevare per primo il prof. Caloi, we fra Italia Centrale c l'Furopa Centrale esiste una discontinuila, mole probabilnente in rorri-pondenzil della Valle Padaná inolue si può dedurre che i milleriali alraversali dalla perlurbazione per raggiungere le sazioni svizzere presentano nua maggiore elaslivila.

Onde $S n$ - Nelle regislrazioni delle stazioni svizzere non è slato powibile individuare con esallezza questo lipo di ondal perlanto lal 
equatione della dromocrona is stalla calcolala con i dali delle cole taxioni italiane erl $i$ ri-ultala:

$$
t_{\mathrm{n}}=\frac{1}{1,41}-0,7
$$

ron le semuenti diflerenze frat i tempi omervali e raleolati:

$\begin{array}{lclc} & t_{0}-t_{c} & & t_{0}-t_{c} \\ \text { Vapoli } & -0,1 & \text { Bolognal } & -1.3 \\ \text { Direnze } & 0,0 & \text { Padova } & 0,1 \\ \text { Pralo } & 0.7 & \text { Priese } & 0.1\end{array}$

11 valore 1.11 kmi-ollemmo per la velocita di questo tipo di onda i in ollimo areordo con quelli ralcolali da altri per l’Ttalia Cenlaile.

Profondita ipoentrale o tempi origine. - Per otlenere la proFondita ipocentrale las appliéalo lat semplice formula

$$
h = 1 \longdiv { ( \kappa T _ { j } ) ^ { 2 } - \widehat { l } ^ { 2 } }
$$

alla -lazione di Romal ollenendo

$$
l=16 \mathrm{~km}^{\mathrm{i}} / \mathrm{al} \text {. }
$$

Helerminala reos la prolondila ipocentrale: dalla relazione

$$
\theta_{11}=1-\frac{1 \Delta^{2}+h^{2}}{V_{P_{g}}}
$$

-o-tiuendo i valori rorrispondenti allat tazione di Roma a ron-ideranclo $J_{p, t}=5.33 \mathrm{~km}=110$ otemmo

rli ron-congenzal

$$
O_{11}=4^{\mathrm{in}} \cdot 2 \cdot 2: 16 \cdot 9
$$

$$
O_{1:}=4 . " 2.211 .50 .
$$

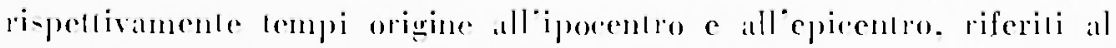
lempo medio delleveropia centrale.

$$
\text { Roma - Istibuto Vazionalo di Crofisica - Igosto } 1948 .
$$

\section{RI. ISSU:TTO}

Si espongono $i$ risuluati dello studio del terremoto di Tramo del 29 gennaio 1913: calcolo delle coordinate epicentrali, delle dromocrone relative alle onde Pog. S. Pn. Pn. Sn. della profondià ipocentrale e dei lempi origine. 


\section{BIBIIOGRAFIA}

Cator P.: Determinazione dolle coordinate ipocrntrali di un terromolo ad origine vicina con i ampi delle onde iongiludinali e trasversali direlle - Ric. Scient.. $19.4,11,4$.

Catot P.: Tempi di tragillo per terremoti ad origine ticina - Rie. Scient., 1939, 11. 5 .

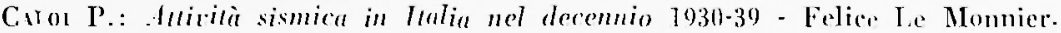
Firme. 